Voix et Images

voixetimages

\title{
Un probable souvenir-écran chez Anne Hébert
}

\section{Henri-Paul Jacques}

Volume 7, numéro 3, printemps 1982

Anne Hébert

URI : https://id.erudit.org/iderudit/200340ar

DOI : https://doi.org/10.7202/200340ar

Aller au sommaire du numéro

\section{Éditeur(s)}

Les Presses de l'Université du Québec

\section{ISSN}

0318-9201 (imprimé)

1705-933X (numérique)

Découvrir la revue

Citer cet article

Jacques, H.-P. (1982). Un probable souvenir-écran chez Anne Hébert. Voix et Images, 7(3), 449-458. https://doi.org/10.7202/200340ar d'utilisation que vous pouvez consulter en ligne.

https://apropos.erudit.org/fr/usagers/politique-dutilisation/ 


\title{
Un probable souvenir-écran chez Anne Hébert
}

\author{
par Henri-Paul Jacques, Université du Québec à Montréal
}

Soit, dans Kamouraska, ce souvenir d'Élizabeth où il est apparemment question de son amant George Nelson:

Si je ferme les yeux, je te retrouve livré aux métamorphoses étranges des mâlés et des hommes. Une image, particulièrement, me poursuit. Tu te souviens de ce coq, dans l'écurie, qui avait pris l'habitude de passer la nuit sur le dos de ton cheval? Un matin. le coq s'est pris les ergots dans la crinière du cheval. Ton cheval se cabre. Se dresse sur ses pattes de derrière. Le coq entravé déploie toute son envergure. Tente de se dégager. A grands coups d'ailes exaspérées. Se débat en vain. Coq et cheval ne forment plus qu'un seul corps fabuleux. Un seul battement, un seul écart d'ailes et de fers. Un seul tumulte, hennissements et cocoricos, emplissent l'écurie de sa clameur, abattant les cloisons de la stalle. Dans un arrachement de plumes et de crins, de planches cassées et de clous tordus.

Je crie. C'est toi, mon amour, cette fureur amputée. Coq et cheval emmêlés, c'est toi, toi courant gaiement à l'épouvante et au meurtre. Sur un dangereux chemin de neige. '

Description tellement vivide qu'elle fait penser à une série de photos accompagnées d'une bande sonore. Et à un souvenir-écran. C'est-à-dire à un phénomène fréquent ainsi défini par la psychanalyse freudienne:

Souvenir infantile se caractérisant à la fois par sa netteté particulière et l'apparente insignifiance de son contenu. Son analyse conduit à des expériences infantiles marquantes et à des fantasmes inconscients. Comme le symptôme. le souvenir-écran est une formation de compromis entre des éléments refoulés et la défense. ${ }^{2}$

Mais, derrière cet écran, que se cache-t-il et simultanément se révèle? Qu'y voir et entendre? Qui?

Comment trouver réponses à ces questions? De la même façon qu'aux énigmes posées par les rêves. Par la décomposition du texte en autant d'éléments qu'il en comporte. Un exemple très simple pour illustrer concrètement la méthode, l'interprétation d'un rève rêvé par une Anglaise et que je traduis partiellement: "Un rêve du nombre 180 me fut interprété au 
cours d'une séance d'analyse avec le sens de $/$ ate nothing. ${ }^{3}$ Ce rêve se réduit en fait a une image visuelle complexe, «180», automatiquement transposés en *one hundred and eighty* dans le récit qu'en fait la rêveuse. Mais cette transposition verbale ne signifie plus rien parce qu'elle efface le rébus de l'image visuelle et oblitère complètement les images acoustiques à partir desquelles le rébus s'est construit. Puisque l'élaboration onirique procède par condensation, le décodage analytique doit procéder en sens inverse et recourir a la décomposition, rétablir l'image visuelle de $\propto 180$ » et la fragmenter en autant d'éléments qu'elle en comporte $(* 1-8.0 \times)$ pour retrouver ensuite les images acoustiques originelles et les représentations de mots d'un certain contenu latent: "/ ate nothing», aje n'ai rien mangé». C'est cette démarche que, mutatis mutandis, j'appliquerai au souvenir-description d'Élizabeth dont je reprendrai chacun des éléments importants pour tenter d'en préciser le sens en me référant chaque fois au corpus hébertien que je considérerai ici comme la somme des associations libres de l'écrivain et le réservoir des variantes de ce probable souvenir-écran.

(1) « métamorphoses étranges des mâles et des hommes *

II y a ici comme des points de suspension à compléter, un vide à remplir par le complémentaire obligé mais inexprimé, "métamorphoses étranges des mâles et des hommes. [des femelles et des femmes]»: simple reprise des propres mots d'Anne Hébert dans un ailleurs de son corpus, « les mâles et les femelles» (E., p. 127)'. "Métamorphoses», changements d'une forme en une autre. Malgré soi, on songe aux Métamorphoses d'Ovide. Et à celles d'Anne Hébert. Ce qui est attribué à des bêtes; mâles et femelles, pourrait bien être le fait d'humains, hommes et femmes, avant transformation: "Les pères et mères viennent aussi nous visiter la nuit. [ . . ] Sous la forme d'un ours brun. Sous l'apparence d'une vache sacrée * (E., p. 51). Et alors, ces "métamorphoses étranges» rendent une tout autre résonance, la résonance de l'étrange, de "l'inquiétante étrangeté" freudienne, du Unheimliche, du connu non reconnu.

(2) * Une image, particulièrement, me poursuit"

Une image! Mot-clé ! Ou une "vision". Celle de sceur Julie de la Trinité qu। affirme son «intention d'user a jamais une image obsédante. [...] Et surtout, ah, surtout! être délivrée du couple sacré» $(E$. p. 7). Rencontre de deux images. l'une persécutrice et l'autre obsédante. Du mème coup, définition précise et sans aucune équivoque du mot-clé, image des parents sexuellement «emmèlés» comme coq. et cheval! À la fois voilée et dévoilée derrière le souvenir-écran d'Élisabeth, image de la scène originaire: 
Scène de rapport sexuel entre les parents, observée ou supposée d'après certains indices et fantasmée par l'enfant. Elle est généralement interprétée par celui-ci comme un acte de violence de la part du père.4

Les deux parents, Adélard et La Goglue, et l'enfant. Les trois de la scène originaire inscrits dans le nom même de sceur Julie de la Trinité. Remarquable manifestation de la double communication, du voilement et du dévoilement simultanés, extraordinaire formation de compromis:

Forme qu'emprunte le refoulé pour être admis dans le conscient en faisant retour dans le symptôme, le rêve, plus généralement toute production de l'inconscient: les représentations refoulées y sont déformées par la défense jusqu'à en être méconnaissables. Dans la même formation peuvent ainsi se satisfaire - en un même compromis - à la fois le désir inconscient et les exigences défensives. ${ }^{5}$

(3) "ce coq $[\ldots]$ sur le dos de ton cheval"

Si ce coq et ce cheval sont vraiment un homme et une femme métamorphosés par les exigences défensives, -ils ne peuvent pas ne pas avoir conservé les traits les plus marquants de leurs formes humaines antérieures. A nous de retrouver chez Anne Hébert une description parallèle d'humains à mettre sur cette description d'animaux. Des humains qui ont mêmes contours que ces animaux. Superposables. Pour reconnaître du connu méconnu. Dans Kamouraska, Louis Clermont et Victoire Dufour!

Élisabeth peut se transporter par la pensée "de place en place» $(K$., p. 207) car elle est "voyante» (K., p. 210) et, à ce titre, "Déjà admise dans l'auberge de Louis Clermont. [ ... ] placée, immobile et silencieuse, au centre de la maison. Afin que je voie tout et que j'entende tout. [...] Dans la salle et dans les chambres. [...] Introduite de force dans l'intimité de cette maison" (ibid.). Puis, régression d'Élisabeth dans le temps, "Le passé franchi, d'un bond prodigieux» (p. 211). Et, elle a examine attentivement [...] obligée, non seulement d'entendre [...], mais forcée de suivre le déroulement des scènes à mesure (p. 213), elle épie longuement un couple au lit (pp. 213-215). Le couple Louis Clermont et Victoire Dufour.

Examinons les contours physiques du coq et de l'aubergiste. Le coq est petit par rapport au cheval, il est «entravé " et il "se débat». Louis Clermont aussi est petit. "sec et maigre» (p. 212), lui aussi est entravé et se débat:

Le petit homme, sec et nerveux, se retient de bouger. S'efforce de paraître calme. $Y$ réussit à peu près comme une anguille vivante attachée à un piquet. Tout son corps au garde-à-vous a soudain des sursauts (p. 214).

Comme le coq, il a aussi pour fonction de réveiller les gens à l'aube puisque. dit-il, «il m'a dit de le réveiller à cinq heures» (p. 219). 
Examinons les contours physiques du cheval et de Victoire Dufour. Si le cheval est bien plus gros que le coq. l'épouse est immense par rapport au mari: «Victoire se déplace lourdement, en roulant des hanches énormes. [...] II se répète, tout attendri, qu'il a épousé la plus grosse» (p. 212). Le cheval «se cabre», il «se dresse sur ses pattes de derrière»: la monture humaine est décrite dans les mèmes termes, $\alpha$ elle së redresse dans son lit, aussi vite que le poids de son corps le lui permet» (p. 213). «Invisible aux yeux de tous» (p. 214) et «Cachée dans cette auberge $\nsim(p .215)$, Elisabeth entend l'inquiétude de Victoire Dufour au sujet de ses congénères:

On entend, de l'autre côté de la cloison, Victoire Dufour qui réveille son mari.

- Vite Clermont, les chevaux se battent dans l'écurie! (p. 219)

Femme-cheval que le mari tente de contrôler, "Tiens-toi donc tranquille, ma femme* (p. 220).

A quoi il faudrait rajouter que le rapport du coq au dos du cheval est fidèlement reproduit dans le rapport du mari au dos de l'épouse, « Son sec petit mari la suit de près (p. 212). A un effet possible de hasard se substitue déjà pour moi un effet certain de vérité grâce au dégagement de ces deux séries d'équations, coq=homme=mari et cheval=femme=épouse. Mais comment valider cette interprétation? II se trouve qu'on peut aboutir exactement au même résultat par une autre méthode. En cherchant, chez Anne Hébert. d'autres associations qui établissent les mèmes équations.

- Or, chez Anne Hébert, le coq réfère à l'homme. Comme dans l'initiation sexuelle du fils Joseph par sa mère Philomène dans Les Enfants du sabbat:

[...] Taureaux, aigles, coqs et serpents sont invoqués comme des dieux, instamment priés de lui prêter leur force virile, afin que s'accomplisse la loi et qu'il couche avec sa mère.

[...]

Le vacarme devient étourdissant.

[...]

Sauve qui peut! Mêlés aux villageois, le départ précipité des dieux offensés, dérangés pour rien. Battements d'ailes, cocoricos, meuglements, sifflements. (E., p. 99)

“Battements d’ailes, cocoricos». Mêmes mots que dans le souvenir-écran. “ coups d'ailes exaspérés. [ ... ] Un seul battement, un seul écart d'ailes [...] cocoricos». Pareillement pour le vacarme étourdissant.

Or, chez Anne Hébert, la femme est à l'occasion comparée et même assimilée au cheval, à la jument, à la pouliche, quand ce n'est pas à l'âne. La putain que fréquente Antoine Tassy ne s'appelle-t-elle pas. comme par hasard, "Horse Marine» $(K .$, pp. 118, 131, 132, 145, 166) ? Et cette description d'une 
servante, $\propto$ Cette longue encolure courbée qu'elle a Florida [ ... ] Un air cheval de corbillard» (K., p. 29). Et cet autoportrait d'Élisabeth, "une croupe dure. Une pouliche de deux ans» (K., p. 10). Ce monologue d'Élisabeth:

L'honneur. La belle idée fixe à faire miroiter sous son nez. La carotte du petit âne. La pitance parfaite au bout d'une branche. Et le petit âne affamé avance $(K .$, p. 9 ).

Cet avatar de la pauvre Florida métamorphosée de cheval en âne, "Florida [...] se dandine, bâtée de légumes et de fruits, pareille à un âne* (K., p. 37). Faudrait-il faire l'hypothese d'un lien subjectif entre le prénom de l'écrivain et le nom de cet animal, «Anne/âne *? A tenir compte des particularités de la prononciation québécoise, la chose ne serait guère surprenante... J'ai gardé pour la fin de ce développement l'assimilation de la mère à la jument. Dans Les Enfants du sabbat, le Dr Painchaud - amoureux malgré lui de scur Julie de la Trinité - s'endort et divague:

Au plus profond de son sommeil il devient très oppressé. Un poids énorme lui écrase la poitrine. [...]

Sœur Julie est assise de tout son poids sur sa poitrine, le chevauchant et lui tournant le dos. [...]

"Je vais mourir étouffé $»$, pense le docteur.

[...]

La voix de soeur Julie, au-dessus de lui maintenant, comme un oiseau invisible.

- Je suis ta night-mère, ta sorcière de la nuit. Tu ne me reconnais donc pas? Je t'emmène avec moi. Je te ferai voir du pays. Et je te monterai à mort, mon pauvre petit cheval idiot. (E., pp. 72-73)

Sa pollution nocturne et cauchemardesque oblige le docteur à changer de pyjama et de draps... On pourrait croire qu'un passage comme celui-ci affaiblit l'interprétation soutenue jusqu'ici, au point de la ruiner. Et pourtant. Pourtant, sceur Julie "chevauchant" le docteur est bien une jument qui chevauche son "pauvre petit cheval idiot». Car, elle le dit explicitement, elle est sa "nightmère», c'est-à-dire sa "night-mare». C'est-à-dire son «cauchemar», C'est-àdire sa "mère-jument» ou, dans notre bilinguisme incestueusement œdipien, sa «mère-mare». Pour faire comprendre ce dont il s'agit ici, je fais remarquer l'identité de l'image acoustique qui élicite simultanément une double représentation de mots: et en français, "mère"; et en anglais, "maren. Je cite ici le rève d'un analysant bilingue:

un rêve dans lequel il avait monté une mare [ "jument"], mot qu'il avait interprété en analyse comme étant mère, ce qui évoquait un souvenir ancien et vague d'avoir été porté sur le dos de sa mère autour de sa chambre d'enfant, sa mère étant à quatre pattes et faisant semblant d'être un cheval: il se rappelait confusément que ce jeu lui avait procuré un immense plaisir. 6 
Quand une Anne est un âne, il n'y a pas à s'étonner outre mesure qu'une jument soit un cheval. D'autant plus que le tiers traumatisé de la scène originaire en conserve un fantasme des deux parents combinés ( «emmèlés\%, dit le souvenir-écran) tout en assumant alternativement les trois positions de la Trinité: celle de l'enfant sidéré, fasciné, hypnotisé, paralysé, inter-pelé (dans cette histoire de peaux) et par l'ouie (Louis Clermont?) et par le regard; celle de la mère-jument agressée et (as)saillie par le père; celle du père-cheval agressant et (as)saillissant la mère, au pas, au trot, ou au galop. Dans la confusion de l'arrière-train des deux en train de forniquer et de geindre. Comme s'ils avaient mat ou se faisaient mal. Alors que c'est l'enfant qui souffre. Ét qui jouit. Car a il y a une chose aussi bruyante que la souffrance, c'est le plaisir»?

Non seulement la femme est-elle occasionnellement comparée ou assimilée au cheval, chez Anne Hébert, mais elle en a encore souvent les attributs. Les CRINS:

Lucie fit couper ses nattes et les porta à son père. Lorsque l'homme vit devant lui la première chevelure tombée, [...] il frappa la fille au visage avec les longs crins noirs empoignés comme des fouets. $(C ., p .36)$

L'ENCOLURE et le POITRAIL: “Cette longue encolure courbée qu'elle a Florida ” (K., p. 29); "la servante penchait vers elle un visage impassible, un poitrail pailleté d'épingles» (C., pp. 151-152). La CROUPE: celle d'Élisabeth, "une croupe dure " $(K .$, p. 10): celle de Philomène-La Goglue, "sa croupe splendide" (E., p. 32), « D'un coup de reins, elle relève sa croupe et s'offre à l'hommage de ses sujets" (ibid., p. 39). "Son ventre et sa croupe s'agitent frénétiquement, d'une façon indécente» (ibid., p. 59). Un CEIL DE CHEVAL, des YEUX DE CAVALE, un REGARD CHEVALIN, à propos d'une seule et mème personne, mère Marie Clotilde la supérieure: «Son bel ceil de cheval» $(E$. p. 21), «Ses gros yeux de cavale affolée" (ibid., p. 61) et "Ses yeux de cavale folle (ibid., p. 179), “son regard chevalin» (ibid., p. 130). Le GALOP: «Ne vous effarouchez pas, mes sceurs. Avant de quitter la pièce au galop" (E., p. 150). La réaction de SE CABRER: à propos d'une servante et de sa "vieille respiration qui s'épuisait et se cabrait» $(C .$, p. 175): à propos d'Élisabeth qui se propose de "Se cabrer au moindre signe de la mort sur te chemin, comme un cheval qui fait demi-tour" ( $K$, p. 97). La BRIDE: «mes petites tantes laissent aller leur Imagination la bride sur le coum (K., p. 99). Dans ce contexte de femme-cheval, j'irais même jusqu'à poser un'rapport et du point de vue phonique et du point de vue morphologıque entre les "pieds-bots" de Mme Mère Tassy dans Kamouraska et les "sabots" de tous ces mammifères onguligrades.

Cette image de femme-cheval est presque toujours une image maternelle, mème quand il s'agit des servantes: Mme Mère Tassy, mère MarieClotilde. Victoire Dufour se retrouve par son prénom et ses initiales dans la lignée des mères sorcières par le biaıs d'une "Victoire Desjardins" (E., p. 103), une des aieules de la sorcière Philomène qui a tenté d'initier sexuellement son fils pour en faire un sorcier: c'est une mère elle aussi, c'est une grosse dont le mari est mince. 


\section{(4) « dans l'écurie»}

Qu'il me suffise ici de citer un passage parallèle:

[...] Ils savent rire et vivre trop fort et s'accouplent dans un vacarme de chats $[\ldots]$

[...] La cabane originelle, avec un seul sac de couchage [...]

Pour peu que l'on ait le courage de regarder à l'intérieur de la cabane, attentif à tous les détails, respirant à pleines bouffées le remugle d'écurie chaude et d'algues pourries qui s'échappe du sac de couchage placé au centre de la pièce. on se rend très bien compte qu'il s'agit ici du lieu d'origine.

Deux géants paisibles dorment, enfermant avec eux, dans leur double chaleur, leurs petits tremblant de froid.

On pourrait se croire à nouveau dans le ventre de la mère gardé par la force du père. Mais lorsque le père livre combat a la mère, il chasse impitoyablement les enfants du sac de couchage.

- Dehors, mes petits maudits!

La bataille entre le père et la mère peut durer toute la nuit. Ou la journée.(E., pp. 85-86)

(5) "un seul corps fabuleux - coq et cheval emmêlés"

Dans les Enfants du sabbat, le frère de Julie épouse une Anglaise, "Cette fille se nomme Piggy. Ce qui signifie en français 'petit cochon's (p. 89). Encore une métamorphose de la femme en animal. La suite du texte définit le mariage, "Ils ne font déjà qu"une seule et même chair" (ibid.). Définition à lire littéralement. Comme variante de la formule du souvenir-écran, "un seul corps fabuleux». Définition inconsciente mais superbe de la scène originaıre même. Anne Hébert reprendra constamment la même définition en référence au coq "entravé " et au cheval entravant. Ainsi, dans Kamouraska: "Cette corde est assez grande pour deux, Elisabeth, ma femme., Les liens du mariage, c'est ca. Une grosse corde bien attachée pour s'étouffer ensemble» (p. 87): «la connivence parfaite qui me lie à cet homme" (p. 72); “Attachée au lit d'un homme fou" (p. 89): "me voici liée à cet homme, dans une seule passion sauvage" (p. 136); "Je veille, liée à cet homme qui dort sous la pluie. Si loin que je sois dans l'espace et le temps. je demeure attachée a George Nelson" (p. 153): "Tous deux liés ensemble dans une seule nécessité" (p. 158). Et auparavant, dans les Chambres de bois: "Je suis liée à un homme" (p. 76); "et cet homme était à moi, et moi à lui, tous deux emmèlés comme deux vignes 
tordues ensemble en une seule tresse (p. 126). Ces mots de la dernière citation, atous deux emmêlés», sont ceux du souvenir-écran, «coq et cheval emmêlés». Mais dans un contexte explicitement sexuel. Rapprochons la "crinière» de la «tresse" et les clous «tordus» des deux vignes atordues".

Notre texte dit bien acoq et cheval ne forment plus qu'un seul corps fabuleux*. Il faudrait étudier dans le détail les fantasmes de condensations fabuleuses, la sirène, la sphinx, le centaure, etc., et les mettre en rapport avec l'image des deux corps embrassés dans l'étreinte sexuelle. Si ce probable souvenir-écran voile et dévoile une scène sexuelle humaine, la métamorphose des protagonistes en animaux révèle du même coup une conception animale de la sexualité humaine. Celle-ci est affaire de bêtes. C'est cela qu'exprime la comparaison qui décrit les retrouvailles d'Élisabèth et de son amant George Nelson après le meurtre du mari, "Nous nous flairons comme des bêtes étrangères $*(K ., p .241)$. Pour l'lnconscient, pas de comparaisons, seulement des assimilations absolues. Donc, à lire selon le code de l'inconscient, les deux amants sont des bêtes et la sexualité humaine est animalité. "Des bêtes étrangères», pourquoi «étrangères»? Ressemblance curieuse avec un autre mot de notre texte, "métamorphoses étranges».

(6) « un seul battement - un seul tumulte - clameur - fureur ameutée*

Sexualité humaine, affaire de bêtes. Affaire d'agressivité aussi, de violence, de sadisme peut-être. Intrication du plaisir et de la douleur, de la libido et de la destrudo. Dans les Chambres de bois: "Michel était devant elle qui venait de lui rendre les coups recus. II l'emmena sur le lit et la posséda avec maladresse et fureur" (p. 116). Dans Kamouraska: "Amour, amour. je te mords, je te bats, je te tue (p. 9); "la violence, l'amour. la dérision [ .. lla nuit, la Petite geint, parfois. De douleur ou de plaisir. Le crime est le même» (p. 100): "Effacer de mon corps toute trace de caresse ou de violencen (p. 117): *Couchée dans le lit d'Antoine, battue par Antoine, caressée par Antoine. ouverte et refermée par Antoıne, violée par Antoine, ravie par Antoine " (p. 129): "Quand on sait ce que "se réconcilier" signifie pour Antoine, il s'agit d'exaucer son désir le plus rapidement possible. Le plus brutalement possible" (p. 146). Dans les Enfants du sabbat, dont il faudrait citer tout le premier chapitre:

Pour sortir de la chambre des enfants, il faut traverser la chambre des parents. Lorsque les noces du père et de la mère se prolongent tout le jour, les enfants se trouvent bloqués dans leur chambre, jusqu'au lendemain.

Sceur Julie éprouve l'angoisse des enfants. Elle se prend à dire, avec la petite fille, ne faisant soudaın plus qu'une seule et même personne avec la petite fille:

- Ca va-t-y finir! Mon Doul Ca va-t-y finir! 
Mais sceur Julie ne peut s'empêcher d'entendre tout le remue-ménage de caresses et de coups qui se passe de l'autre côté de la cloison. (p. 10)

J'ai déjà cité le a vacarme de chats [... ]Lorsque le père livre combat a la mère» (p. 85) ainsi que «La bataille entre le père et la mère» (ibid.). Je ne cıterai pas les bourrades d'Adélard et de Philomène (p. 96). Mais je citerai encore la relation sexuelle incestueuse d'Adélard avec Julie:

Philomène est couchée sur le dos, toute seule dans le noir.

Derrière la cloison, les cris de la fille, le rire du père. Ce qu'ils font ensemble, tous les deux, couchés sur la paillasse. Ce que le père n'obtient que par la force. Ce que la fille apprend à défendre, puis à désirer, a aimer, aux portes de la mort. L'enchantement de la violence. La fille se débat, griffe et mord, hurle. jusqu'à ce que l'enfer la secoue de bonheur et la laisse comme morte sur la paille. (p. 110)

L'enfer secouant Julie. Pour Élisabeth, c'était la géhenne, G.N., son amant George Nelson; c'était aussi un athée, son mari Antoine Tassy. Par un juste retour des choses, la scène originaire jadis subie par la fille est maintenant imposée à la mère dans cette permutation de leurs rôles. Rapprochons des "cloisons de la stallen du souvenir-écran ces deux allusions à "l'autre côté de la cloison " et à " Derrière la cloison”. Comme il faut rapprocher les "planches cassées * du souvenir d'Élisabeth de cet autre passage où il est question d'elle. «La fatigue de l'amour l'accable et lui casse les reins* (K., p. 167)

Est-il si difficile maintenant de lire autrement que comme un simple cliché innocent ale lit en bataille» (K., p. 116), même si le contexte immédiat n'y est pas explicitement sexuel? A chacune de faire ou, bien entendu, de défaire son lit comme elle l'entend.

Est-il besoin de gloser un passage comme celui-ci:

Un homme s'acharne, à coups de crosse de pistolet sur un mort çouché, la face dans la neige. Il frappe jusqu'à l'usure de la force surhumaine en lui déchainée. Maitre de la vie et de la mort. Un instant le vainqueur essuie son visage sur sa manche. Cherche dans son cour la femme pour laquelle... Désire s'accoupler immédiatement avec elle. Triomphalement. Avant que ne déclinent sa puissance et sa folie. (K., p. 234)

Quel plus bel exemple trouver de l'intrication de l'amour et de la mort?

\section{CONCLUSION}

Chacun des mots importants du texte de ce souvenir-écran qui semble ne décrire qu'une simple violence animale accidentelle se lit dans des passages parallèles dont plusieurs décrivent une relation sexuelle. 
Comment parler de hasards et de coïncidences? Anne Hébert ne nous donne-t-elle pas à lire ici un remarquable dérivé littéraire du traumatisme de la scène originaire? A quoi aucun humain n'échappe. A quoi tout humain doit toute sa vie revenir. Bénéfique traumatisme dont il faut constater la présence et la puissance dans l'ordre de la pulsion créatrice. Dans l'ordre de la Pulsion.

Mais pourquoi me suis-je introduit d'un rêve rêvé par une Anglaise pour illustrer le processus de la décomposition? Serait-ce parce que «Vous parlez en langue étrangère, docteur Nelson» (K., p. 248) et que je veux laisser entendre - d'une autre oreille - que l'image acoustique du "coq» gaulois sur le dos de votre cheval dit un autre mot dans votre langue maternelle? Sans doute. Gauloiserie gratuite? Non. Car, inconsciemment ou non, votre "cheval se retrouve inopinément en anglais dans le nom de la putain «Horse Marine»: complémentaires obligés; comme les initiales des deux rivaux, A.T. et G.N. Serait-ce parce que ce souvenir-écran s'introduit deux fois d'un si beau «Farewell my love» (K., p. 191)?

En finissant.

Qu'on me permette.

De dédier ce texte à J.S. dans la crinière de qui je me suis pris les ergots. Parce que "Si je ferme les yeux, je te retrouve» $(K .$, p. 191). Parce que...

1. Anne Hébert, Kamouraska, Paris, Les Éditions du Seuil, 1970, p. 191. Pour les références dans le reste de mon analyse, j'utiliseraı les abréviations suivantes:

K. Kamouraska.

C. Les Chambres de bois, Paris, Les Éditions du Seuil, 1958

E. Les Enfants du sabbat, Paris, Les Editions du Seuil, 1975

2. J. Laplanche et J.-B. Pontalis, Vocabulaire de la psychanalyse (1967), Paris, Presses Universitaires de France. 1981. s.v.

3. Ella Freeman Sharpe, Dream Analysis (1937). London, The Hogarth Press Ltd and The Institute of Psycho-Analysis, 1951, p. 92.

4. J. Laplanche et J.-B. Pontalis, op. cit., s.v.

5. Ibid., s.v.

6. J.-C. Flugel, "A Case of Affective Inhibition of an Intellectual Process", p. 115 in The International Journal of Psycho-Analysis, vol. 4, 1923.pp. 111-117. Pour mieux faire appréhender de quoi il retourne dans ce jeu de mots et dans cette histoire de cauchemar, je réfère à l'ouvrage classıque de Ernest Jones. On the Nightmare (1931). New York, Liverıght Paperbound Edition, 1971; cet ouvrage est maintenant accessible en traduction chez Payot.

7. Marcel Proust, Sodome et Gomorrhe, Parıs, Gallimard, tome II. p. 609. 